\title{
CONVERGENCE OF SEMI-DISCRETE DIFFERENCE SCHEMES OF ABSTRACT CAUCHY PROBLEMS
}

\author{
HARUO SUNOUCHI
}

(Received August 18, 1969)

\begin{abstract}
1. Introduction. It is well-known that the Cauchy problem and difference approximations of its solution are closely related to the theory of semigroups, but these relations are usually treated under the assumption that the semigroup generated by the solution operators of the Cauchy problem is a $\left(C_{0}\right)$-semigroup. Trotter [9] shows that the difference approximations for the Cauchy problem is formulated in terms of semigroups and reduces the problem to the convergence of semigroups. On the other hand, the theory of semigroups is developed in more general classes, for example, in the class of $(A)$ or $(0, A)$. Phillips [8] formulates the abstract Cauchy problem in terms of $\left(C_{0}\right)$ - and $(0, A)$-semigroups. The theory of convergence of semigroups is generalized to $(1, A)$-semigroups by Miyadera [6], and to $(A)$ - and $(0, A)$-semigroups by Oharu and Sunouchi [7].

The purpose of this paper is to formulate the semi-discrete difference approximations to Cauchy problem in terms of semigroups. In $\$ 2$, we show that the problem of convergence of semi-discrete difference approximations of Cauchy problem is reduced to the problem of convergence of semigroups. In order to treat this problem by semigroup-theoretic approach, we shall introduce the notion of well-posedness in the sense of semigroup and study some relations between the abstract Cauchy problem and its semigroup. In this formulation our results on the convergence of $(A)$ - and $(0, A)$-semigroups are applicable to the problem. But if we assume that the Cauchy problem is well-posed in the sense defined in $\S 2$, then the conditions of convergence are given in very simple forms (\$3). As an application, in the case of constant coefficients considered in $L^{2}\left(R^{d}\right)$, we can show there are always semi-discrete difference approximations which converge to the solution of the given Cauchy problem (in \$4). Finally, in this respect, we shall show some examples of $(0, A)$-semigroups generated by the solution operators of Cauchy problems in simple forms (in the Appendix). These examples will be interested in themselves.
\end{abstract}

2. Abstract Cauchy problem and semi-discrete difference scheme. Let us consider the abstract Cauchy problem 


$$
\frac{d u}{d t}=A u, \quad u(0)=u_{0},
$$

in a Banach space $X$. Here $A$ is a densely defined closed linear operator. We shall assume that $A$ has a core, that is, a linear dense set contained in the domain $D\left(A^{\infty}\right)=\cap D\left(A^{n}\right)$, such that the closure of $A$ restricted to $D$ is equal to $A$ and $A(D) \subset D$. This condition is usually satisfied by differential operators. (Concerning the notion of core and its properties, refer Kato [4].)

The process of setting up a sequence of finite difference approximations to the Cauchy problem (2.1) may be described in the following general terms. Let $\left\{A_{n}\right\}$ be a sequence of bounded linear operators which approximates $A$ in the sense that

$$
A_{n} u \rightarrow A u, \quad \text { for } u \in D,
$$

then we obtain the sequence of equations

$$
\frac{d u_{n}}{d t}=A_{n} u_{n}, \quad u_{n}(0)=u_{0},
$$

which will be called a semi-discrete difference scheme. (2.2) will be called a consistency condition. The solution of (2.3) for each $n$ is obviously represented as

$$
u_{n}(t)=\exp \left(t A_{n}\right) u_{0}=T_{n}(t) u_{0} .
$$

Here $T_{n}(t)=\exp \left(t A_{n}\right)=\sum_{k=0}^{\infty}\left(t A_{n}\right)^{k} / k !$, which is well-defined, because $A_{n}$ is a bounded linear operator. Thus we obtain a sequence of uniformly continuous semigroups $\left\{T_{n}(t)\right\}$. The general descriptions of semi-discrete difference approximations for constant coefficient cases are given by Birkhoff and Varga [1] in some details. See also $\$ 4$.

Now the problem of semi-discrete approximations is: under what conditions do the approximate solutions $u_{n}(t)=T_{n}(t) u_{0}$ converge to the solution $u(t)$ of $(2.1)$ for $t>0$ or $t \geqq 0$ ?. Note that the problem is formulated whether or not the Cauchy problem (2.1) is well-posed.

Trotter [9] proved (in a more general form) that, under the consistency condition, if there are constants $M$ and $\omega$ such that

$$
\left\|T_{n}(t)\right\|=\left\|\exp \left(t A_{n}\right)\right\| \leqq M e^{\omega t},
$$

and the resolvent set of $A$ intersects the half plane $\{\lambda ; \operatorname{Re} \lambda>\omega\}$, then the Cauchy problem (2.1) is well-posed in the sense that the solution operators 
'T(t), defined by the solutions $u(t)=T(t) u_{0}$, form a $\left(C_{0}\right)$-semigroup, and the sequence of approximate solutions $u_{n}(t)$ converges to $u(t)$ strongly, and uniformly in $t$ in any finite interval of $[0, \infty)$.

However, as will be shown presently, there are Cauchy problems which are well-posed in weaker sense. We shall restrict our attentions to the well-posedness which can be defined in terms of semigroup theory.

A function $u(t)=u\left(t ; u_{0}\right)$ is called a.genuine solution of (2.1), if

i ) $u(t)$ is strongly absolutely continuous and continuously differentiable in each finite interval of $(0, \infty)$,

ii) $u(t) \in D(A)$ and $\frac{d u(t)}{d t}=A u(t)$, for each $t>0$,

iii) $u\left(t ; u_{0}\right) \rightarrow u_{0}, \quad$ as $t \rightarrow 0+$.

The Cauchy problem (2.1) is said to be well-posed in the sense of semigroup (S.G. well-posed) if there is a family of bounded linear operators $T(t)$, $t>0$, defined on the core $D$ of $A$, such that for any $u_{0} \in D(2.1)$ has a unique genuine solution $u(t)=T(t) u_{0} \in D$ for all $t>0$.

We note here that if $u(t)$ is a genuine solution, then $d u(t) / d t$ is continuous for $t>0$ and Bochner integrable in any finite interval of the form $(0, \beta)$.

When the Cauchy problem is S.G. well-posed, we can of course extend the definition of $T(t)$ to a bounded linear operator defined on the whole space $X$ by closure; this extended operator will be denoted by the same symbol $T(t)$ and called the solution operator of (2.1).

THEOREM 1. If the Cauchy problem (2.1) is S.G. well-posed, then the solution operators $\{T(t)\}$ form a semigroup, that is,

$$
\begin{gathered}
T(s+t)=T(s) T(t), \quad s, t \geqq 0, \\
T(t) u \rightarrow T\left(t_{0}\right) u, \quad \text { as } t \rightarrow t_{0} \text { for any } t_{0}>0, u \in X .
\end{gathered}
$$

Further we obtain

$$
\frac{d}{d t} T(t) u_{0}=A T(t) u_{0}=T(t)\left(A u_{0}\right) \quad \text { for } t \geqq 0, u_{0} \in D
$$

ProOF. (2.6) is obvious. (2.7) follows from the strong continuity of $\left\{T(t) u_{0} ; u_{0} \in D\right\}$, denseness of $D$ and the well known theorem of semigroups concerning strong measurability and strong continuity ([3; Theorem 10.2.3.]).

Since we have assumed that $u_{0} \in D$ implies $A u_{0} \in D, T(t)\left(A u_{0}\right)$ is also a 
genuine solution. Therefore $A\left[T(t)\left(A u_{0}\right)\right]=\frac{d}{d t} T(t)\left(A u_{0}\right)$ is Bochner integrable in $(0, \beta)$ and

$$
\begin{aligned}
T(t)\left(A u_{0}\right) & =A u_{0}+\int_{0}^{t} \frac{d}{d t} T(t)\left(A u_{0}\right) d t \\
& =A u_{0}+\int_{0}^{t} A\left[T(t)\left(A u_{0}\right)\right] d t \\
& =A u_{0}+A\left[\int_{0}^{t} T(t)\left(A u_{0}\right) d t\right] .
\end{aligned}
$$

Put $z(t)=u_{0}+\int_{0}^{t} T(t)\left(A u_{0}\right) d t$, then $z(t)$ is strongly continuously differentiable for $t \geqq 0$ and $d z(t) / d t=T(t)\left(A u_{0}\right)=A(z(t))$. Thus $z(t)$ is also a genuine solution of (2.1), and $z(t)=T(t) u_{0}$ by unicity. Now (2.8) is obvious.

As mentioned in $\S 1$, the Cauchy problem is usually treated under the assumption that the solution operators form a $\left(C_{0}\right)$-semigroup or more stronger, holomorphic semigroup. But some Cauchy problems of parabolic systems in the sense of Shilov generate semigroups of class $(0, A)$, which will be shown by examples in the appendix. We shall say the Cauchy problem (2.1) is $(A)$-, $(0, A)$ - or $\left(C_{0}\right)$-rell-posed if the semigroup of the solution operators is $(A)$-, $(0, A)$ - or $\left(C_{0}\right)$-semigroup, respectively.

We close this section with a summary of semigroup theory and a theorem, but we assume familiarity with these basic facts; see Hille-Phillips [3].

Let $\{T(t) ; t>0\}$ be a semigroup, then the type of $\{T(t)\}$ is defined by

$$
\omega_{0}=\lim _{t \rightarrow \infty} t^{-1} \log \|T(t)\|<\infty .
$$

A semigroup $\{T(t)\}$ is said to be of class (A) if $X_{0}=\bigcup_{t>0} T(t)[X]$ is dense in $X$, and if there is an $\omega_{1}>\omega_{0}$ such that for any $\lambda$ with $\operatorname{Re} \lambda>\omega_{1}$, there is a bounded linear operator $R(\lambda)$ such that

a)

b)

c)

$$
R(\lambda) u=\int_{0}^{\infty} e^{-\lambda t} T(t) u d t, \quad \text { for } u \in X_{0}
$$

$$
\sup \left\{\|R(\lambda)\| ; \operatorname{Re} \lambda>\omega_{1}\right\}<\infty
$$

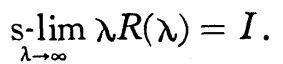


Further, if $\int_{0}^{1}\|T(t) u\| d t<\infty$, then it is said to be of class $(0, A)$. $\left(C_{0}\right)$ semigroup is defined as follows: $\lim _{t \rightarrow 0+} T(t) u=u$ for each $u \in X$; it then follows that, given $\omega>\omega_{0}$, there exists an $M>0$ such that $\|T(t)\| \leqq M e^{\omega t}$, for $t \geqq 0$. For semigroups of any class mentioned above,

$$
\lim _{t \rightarrow 0+} t^{-1}(T(t)-I) u=A^{0} u,
$$

exists for a set $D\left(A^{0}\right)$, dense in $X$ and $A^{0}$ is preclosed. ( $A^{0}$ is closed for $\left(C_{0}\right)$-case.) Then resolvent $R(\lambda ; A)$ of the closure $A$ of $A^{0}$ exists for $\operatorname{Re} \lambda>\omega_{1}$ and $R(\lambda)$ $=R(\lambda ; A)$. The followings will be used: if $u \in D(A)$, then

$$
\frac{d(T(t) u)}{d t}=A T(t) u=T(t) A u \quad \text { for } t>0,
$$

([3; Theorem 11.5.3.]), and if $T(t)$ is of $(0, A)$-class, then

$$
\lim _{t \rightarrow 0+} T(t) u=u, \quad \text { for } u \in D(A),
$$

([3; Theorem 11.5.4.]).

Finally, the following theorem will clarify the relation between the Cauchy problem and its semigroup.

THEOREM 2. When the Cauchy problem (2.1) is (A)-well-posed, then A is the infinitesimal generator of the semigroup of solution operators.

ProOF. By the definition of $(A)$-semigroup, the bounded linear operator $R(\lambda)=\left(\lambda I-A^{\prime}\right)^{-1}$ is defined for sufficiently large $\operatorname{Re} \lambda$, and

$$
R(\lambda) u=\int_{0}^{\infty} e^{-\lambda t} T(t) u d t
$$

for $u$ where $T(t) u \rightarrow u$ as $t \rightarrow 0$. ( $A^{\prime}$ is the infinitesimal generator of $\{T(t)\}$.) Now (2.9) has a meaning for $u_{0} \in D$ and, employing (2.8), we obtain

$$
\begin{aligned}
R(\lambda) A u_{0} & =\int_{0}^{\infty} e^{-\lambda t} T(t)\left(A u_{0}\right) d t \\
& =\int_{0}^{\infty} e^{-\lambda t} \frac{d}{d t}\left(T(t) u_{0}\right) d t \\
& =-u_{0}+\lambda R(\lambda) u_{0},
\end{aligned}
$$


that is, $R(\lambda)(\lambda I-A) u_{0}=u_{0}$ for $u_{0} \in D$. Similarly we obtain $(\lambda I-A) R(\lambda) u_{0}=u_{0}$ for $u_{0} \in D$. But then we know $R(\lambda)=(\lambda I-A)^{-1}$, by using the closedness of $A$ and denseness of $D$. Therefore $(\lambda I-A)^{-1}=\left(\lambda I-A^{\prime}\right)^{-1}$, and $A=A$.

3. Convergence of semi-discrete difference schemes. As mentioned before, the convergence of semi-discrete difference schemes is reduced to the convergence of semigroups $\left\{T_{n}(t)\right\}$. Recently we obtain a theorem on convergence of semigroups of class $(A)$ or $(0, A)$ and the results will be applicable as a criterion for convergence of semi-discrete difference schemes of Cauchy problems. This may be stated as follows:

THEOREM 3. Under the consistency condition, suppose that

(3.1) there is a $\gamma>0$ such that

$$
\begin{gathered}
\sup _{n} \int_{0}^{\infty} e^{-\gamma t}\left\|\exp \left(t A_{n}\right) u\right\| d t<\infty \quad \text { for each } u \in X, \\
\left\|\lambda R\left(\lambda ; A_{n}\right)\right\| \leqq M, \quad \text { for } \lambda>\gamma, \\
\sup _{n}\left\|\exp \left(t A_{n}\right)\right\|<\infty \quad \text { for each } t>0, \\
\rho(A) \cap\{\lambda ; \operatorname{Re} \lambda>\gamma\} \neq \emptyset,
\end{gathered}
$$

then the Cauchy problem (2.1) is well-posed in the sense of $(0, A)$, and $u_{n}(t) \rightarrow u(t)$, strongly, and uniformly in any finite closed interval of $(0, \infty)$.

If (3.1) is replaced by

(3.1)' there are real numbers $\gamma$ and $L>0$ such that

$$
\left\|R\left(\lambda ; A_{n}\right)\right\| \leqq L \quad \text { for } \operatorname{Re} \lambda>\gamma
$$

then the Cauchy problem is (A)-well-posed.

Proof is rather complicated because the theorem is strong enough to imply the well-posedness of the Cauchy problem by means of the semi-discrete approximations. See, Oharu and Sunouchi [7; Theorems $(1, A),(A)$ and their Corollary 1].

REMARK. It is obvious from (3.1) that $R\left(\lambda ; A_{n}\right)=\int_{0}^{\infty} e^{-\lambda t} \exp \left(t A_{n}\right) d t$ is well-defined for $\operatorname{Re} \lambda>\gamma$, therefore $\{\lambda ; \operatorname{Re} \lambda>\gamma\} \subset \rho\left(A_{n}\right)$. Thus we know, with the consistency condition, 
(3.5) $R\left(\lambda ; A_{n}\right) \rightarrow R(\lambda ; A)$, and the range of $R(\lambda ; A)$ is dense in $X$.

(cf. Kato [4; Theorem VIII. 1.5]). These conditions are due to Trotter [9].

It is sometimes (or usually) known that the Cauchy problem is well-posed in either sense mentioned above and the problem is only to show the convergence of semi-discrete approximations. (A condition of $(0, A)$-well-posedness is given by Phillips [8].)

THEOREM 4. Let us assume the Cauchy problem (2.1) is $(0, A)-((A)-$, or $\left.\left(C_{0}\right)^{-}\right)$well-posed, and the type of $\{T(t)\}$ is $\omega_{0}$. If the consistent semidiscrete scheme (2.3) satisfies the conditions

(S) there are real numbers $\gamma\left(>\omega_{0}\right)$ and $L>0$ such that

$$
\begin{aligned}
\left\|R\left(\lambda ; A_{n}\right)\right\| & \leqq L \quad \text { for } \operatorname{Re} \lambda>\gamma, \\
\sup _{n}\left\|\exp \left(t A_{n}\right)\right\| & <\infty, \quad \text { for each } t>0,
\end{aligned}
$$

then $u_{n}(t) \rightarrow u(t)$, strongly for $t>0$, and the convergence is uniform in $t$ for any compact interval of $(0, \infty)$.

PROOF. Put $f(t)=\sup _{n}\left\|T_{n}(t)\right\|<\infty$, then $f(t)$ is non-negative, measurable and submultiplicative, so that

$$
f(t) \text { is bounded in any finite interval of the form }[\varepsilon, 1 / \varepsilon] \text {, }
$$

$$
\lim _{t \rightarrow \infty} t^{-1} \log f(t)<\infty
$$

([3; VIII, §7.4]). Hence, for each $\varepsilon>0$, there is a $\mu_{0}$ such that

$$
\sup _{n}\left\|\exp \left(-\mu_{0} t\right) T_{n}(t)\right\| \leqq 1, \quad \text { for } t \geqq \varepsilon .
$$

Therefore the problem is reduced to the case of $T_{n}(t)$ and $T(t)$ of negative type, by considering equivalent semigroups $\exp (-\mu t) T_{n}(t)$ and $\exp (-\mu t) T(t)$, where $\mu=\max \left(\mu_{0}, \gamma\right)$.

Then we know the estimates

$$
\left\|\exp \left(t A_{n}\right) u-\left(I-t A_{n} / k\right)^{-k} u\right\| \leqq \frac{t^{2}}{k} K(u), \quad \text { for any } u \in X
$$

and

$$
\left\|T(t) u-(I-t A / k)^{-k} u\right\| \leqq \frac{t^{2}}{k} K(u), \quad \text { for } u \in D\left(A^{4}\right)
$$


(cf. [7 ; Lemma 5]). Thus we obtain, for $u \in D\left(A^{4}\right)$,

$$
\begin{aligned}
\| T_{n}(t) u & -T(t) u\|\leqq\| T_{n}(t) u-\left(I-t A_{n} / k\right)^{-k} u \| \\
& +\left\|\left(I-t A_{n} / k\right)^{-k} u-(I-t A / k)^{-k} u\right\|+\left\|(I-t A / k)^{-k} u-T(t) u\right\| \\
\leqq & \frac{2 t^{2}}{k} K(u)+\left\|\left(I-t A_{n} / k\right)^{-k} u-(I-t A / k)^{-k} u\right\|,
\end{aligned}
$$

hence, by (3.5),

$$
\lim \sup \left\|T_{n}(t) u-T(t) u\right\| \leqq \frac{2 t^{2}}{k} K(u)
$$

Now we obtain, as $k \rightarrow \infty, T_{n}(t) u \rightarrow T(t) u$ for $u \in D$. Since $D$ is dense in $X$ and $\sup \left\|T_{n}(t)\right\|<\infty$, we obtain the result.

The uniformity of the convergence follows from (3.6) and equicontinuity of $T_{n}(t) x$ in $[\varepsilon, 1 / \varepsilon]$. But this will be obvious.

4. Constant coefficient cases. In this section we shall consider the Cauchy problem with constant coefficients, that is,

$$
\frac{d u}{d t}=P(D) u, \quad u(0)=u_{0}(x),
$$

in a Banach space $X$, where $P(D)$ is a partial differential operator with respect to $x \in R^{d}$ with constant $N \times N$ matrix coefficients, and $u(t)=u(x, t)$ is an $N$ dimensional vector. General descriptions of semi-discrete approximations for constant coefficient cases are given by Birkhoff and Varga [1] in some details. Following their arguments, let $h=\left(h_{j}\right), h_{j}>0$ be a vector whose components specify the mesh spacings, and approximate each partial derivative $D^{l} u_{j}=D_{1}^{l_{1}} \ldots$ $D_{d}^{l_{d}} u_{j}, D_{k}=i \frac{\partial}{\partial x_{k}}$, by a divided difference of the form

$$
\left(\Pi h_{k}^{-l_{k}}\right) \sum_{m} \mu_{m}^{(l)} u_{j}\left(x_{1}+m_{1} h_{1}, \cdots, x_{d}+m_{d} h_{d}\right)=\delta_{h}^{l}\left[u_{j}\right]
$$

where the $\mu_{m}^{(l)}$ are fixed constant independent of $h$. The assignment of a particular choice of a set of such divided difference approximations (4.2) to each $D^{l}$ in (4.1) will be called a semi-discrete finite difference scheme $\Delta$. When both the scheme $\Delta$ and the mesh vector $h$ are specified, we will speak of a semidiscretization of a system (4.1), and denote the resulting approximation to $P(D)$ by $\Pi(\Delta, h)$. Then $\Pi(\Delta, h)$ is a linear combination of translation operators, and so a bounded linear operator. It will be natural to require that

$$
\Pi(\Delta, h) u \rightarrow P(D) u, \text { for } u \in D,
$$


which is the consistency condition. We obtain a sequence of approximating equations :

$$
\frac{d u_{h}}{d t}=\Pi(\Delta, h) u_{h}, \quad u_{h}(0)=u_{0} .
$$

The abstract formulation of this setting is given in $\S 2$.

Let us now consider the problem in $L^{2}\left(R^{d}\right)$, that is, the norm is defined by

$$
\|u(t)\|=\left(\int|u(t, x)|^{2} d x\right)^{1 / 2}=\left(\int \sum_{j=1}^{N}\left|u_{j}(t, x)\right|^{2} d x\right)^{1 / 2} .
$$

Defining the Fourier transform by

$$
\hat{u}(\xi)=(2 \pi)^{-d / 2} \int u(x) \exp (-i<x, \xi>) d x
$$

then (4.1) reduces (formally) to the following Cauchy problem for a system of ordinary differential equations with $\xi$ as a parameter, namely,

$$
\frac{d \hat{u}}{d t}=P(\xi) \hat{u}(\xi, t), \quad \hat{u}(\xi, 0)=\hat{u}_{0}(\xi),
$$

and (4.4) to

$$
\frac{d \hat{u}_{h}}{d t}=A\left(\xi, \Pi_{h}\right) \hat{u}_{h}, \quad \hat{u}_{h}(0)=\hat{u}_{0}
$$

$A\left(\xi, \Pi_{h}\right)$ is called the amplification matrix.

The solution of (4.5) is

$$
\hat{u}(\xi, t)=\exp (t P(\xi)) \hat{u}_{0}(\xi),
$$

for any initial function $\hat{u}_{0}(\xi)$ if it has a compact support, and the solution of (4.6) is given by

$$
\hat{u}_{h}(t, \xi)=\exp \left(t A\left(\xi, \Pi_{h}\right)\right) \hat{u}_{0}(\xi)
$$

for any $\hat{u}_{0}$.

Now if the centered difference quotient:

$$
\frac{\delta u_{j}}{\delta x_{k}}=\frac{u_{j}\left(x+h_{k} e_{k}\right)-u_{j}\left(x-h_{k} e_{k}\right)}{2 h_{k}},
$$


( $\mathrm{e}_{k}$ is the $k$-th unit vector) is used as an approximation of $\left(\partial u_{j} / \partial x_{k}\right)$, then the entries of the amplification matrix $A\left(\xi, \Pi_{h}\right)$ are exactly same as the corresponding ones of the given matrix $P(\xi)$, where $\xi_{k}$ is replaced by $\xi_{k}(h)=\sin \left(h_{k} \xi_{k}\right) / h_{k}$. This is easily seen by

$$
\frac{\widehat{\delta u_{j}}}{\delta x_{k}}(\xi)=\frac{\sin \left(h_{k} \xi_{k}\right)}{h_{k}} \hat{u}_{j}(\xi)
$$

THEOREM 5. Assume the Cauchy problem (4.1) with constant coefficient matrices is well-posed in any one of $(A)-,(0, A)-$, and $\left(C_{0}\right)$-senses in $L^{2}\left(R^{d}\right)$, then there is a semi-discrete finite difference scheme which converges to the solution.

Proof. By theorem 4, it is sufficient to show the conditions (S) and (B). If we use the centered difference approximations (4.9), then $A\left(\xi, \Pi_{h}\right)=P(\xi(h))$. We note that $\left|\xi_{k}\right| / 2 \leqq\left|\xi_{k}(h)\right| \leqq\left|\xi_{k}\right|$ for $\left|\xi_{k}\right| \leqq \pi / 2 h_{k}$, then

$$
\begin{aligned}
& \sup _{\xi(h)}\left|(\lambda I-P(\xi(h)))^{-1}\right| \leqq \sup _{\xi}\left|(\lambda I-P(\xi))^{-1}\right|, \\
& \sup _{\xi(h)}|\exp (t P(\xi(h)))| \leqq \sup |\exp (t P(\xi))|,
\end{aligned}
$$

and the right hand sides are independent of $h$, we have (S) and (B), and the proof is completed.

APPENDIX. Some examples of $(0, A)$-well-posedness.

Let us consider the Cauchy problem with constant coefficients :

$$
\frac{d u}{d t}=P(D) u, \quad u(0)=u_{0} \quad \text { in } L^{2}\left(R^{d}\right) .
$$

Since $P(D)$ is preclosed in $L^{2}$, we obtain $P_{0}(D)$, called the minimal operator of $P(D)$, by taking the closure of $P(D)$. If we take differentiation $P(D)$ as distribution sense, we obtain the maximal operator $P_{1}(D)$ with the domain $D\left(P_{1}(D)\right)=\left\{u \in L^{2} ; P(D) u \in L^{2}\right\}=\left\{\hat{u} \in L^{2} ; P(\xi) \hat{u}(\xi) \in L^{2}\right\}$. It is obvious that $P_{1}(D) \supset P_{0}(D)$, but we are considering these operators in $L^{2}$ on the whole $R^{d}$, it is well-known that $P_{1}(D)=P_{0}(D)$, which will be denoted by $P(D)$. Then we obtain the abstract Cauchy problem

$$
\frac{d u}{d t}=P(D) u=A u, \quad u(0)=u_{0}, \quad \text { in } L^{2}\left(R^{d}\right),
$$

and as a core we may take $D=\{u \in S ; \hat{u}(\xi)$ has a compact support. $\}$.

Since the Fourier transform preserves the Hilbert space structure of $L^{2}$, we 
may consider the problem in the transformed space $L^{2}\left(\widehat{R}^{d}\right)$, see, (4.5). The correspondence given by the Fourier transform will be summarized as follows:

$$
\begin{aligned}
& P(D)=A \longleftrightarrow P(\xi), \\
& T^{\prime}(t) \longleftrightarrow \exp (t P(\xi)),
\end{aligned}
$$

and $\|T(t)\|=\sup \left\{|\exp (t P(\xi))| ; \xi \in \widehat{R}^{d}\right\}$,

$$
(\lambda I-A)^{-1} \longleftrightarrow(\lambda I-P(\xi))^{-1},
$$

when either side is bounded, and then $\left\|(\lambda I-A)^{-1}\right\|=\sup \left\{\left|(\lambda I-P(\xi))^{-1}\right| ; \xi \in \widehat{R}^{d}\right\}$.

REMARK. If the Cauchy problem (A.2) is S.G. well-posed, then the type of semigroup: $\omega_{0}=\lim t^{-1} \log \|T(t)\|$ is finite. Let $\lambda_{j}(\xi)$ be the eigenvalues of $P(\xi)$ and put $\Lambda(P)=\sup \left\{\max _{j} \operatorname{Re} \lambda_{j}(\xi) ; \xi \in \widehat{R}^{d}\right\}$, then $\|T(t)\| \geqq \exp (t \Lambda(P))$, and so

$$
\omega_{0} \geqq \Lambda(P) \text {. }
$$

This implies, if the Cauchy problem is S.G. well-posed, then it is well-posed in the sense of Petrowski.

We note here that the condition of $\left(C_{0}\right)$-well-posedness is, as easily seen, $\sup \{\|T(t)\| ; 0 \leqq t \leqq T\}<\infty$ for any $T>0$, and the algebraic condition for $P(\xi)$ is given by Kreiss [5].

Before entering examples, we note

LEMMA A. Let $\lambda \in \rho(A)$, then we obtain a representation of the resolvent of $A$ by

$$
(\lambda I-A)^{-1} u=\int_{0}^{\infty} \exp (-\lambda t) T(t) u d t \quad \text { for } u \in D
$$

PROOF. For any fixed $\xi$ such as $\lambda I-P(\xi)$ is regular,

$$
(\lambda I-P(\xi))^{-1} \hat{u}(\xi)=\int_{0}^{\infty} \exp (-\lambda t) \exp (t P(\xi)) \hat{u}(\xi) d t
$$

follows from the matrix theory. Now $\lambda \in \rho(A)$ implies this equation is valid for each $\xi$ and

$$
(\lambda I-A)^{-1} u \longleftrightarrow(\lambda I-P(\xi))^{-1} \hat{u}(\xi)
$$




$$
=\int_{0}^{\infty} \exp (-\lambda t) \exp (t P(\xi)) \hat{u}(\xi) d t
$$

in $L^{2}$. On the other hand, if $u \in D$, then by the Fubini theorem, the inverse Fourier transform of $\int_{0}^{\infty} \exp (-\lambda t) \exp (t P(\xi)) \hat{u}(\xi) d t$ equals to $\int_{0}^{\infty} \exp (-\lambda t) T(t) u d t$, thus we obtain the representation.

REMARK. $\lambda \in \rho(A)$ implies that the operator $R(\lambda)$ defined by

$$
R(\lambda) u=\int_{0}^{\infty} \exp (-\lambda t) T(t) u d t, \text { for } u \in D
$$

is a bounded linear operator, and $R(\lambda)=R(\lambda ; A)$.

In order to verify the condition a) of the definition of $(A)$-semigroups, we have to show (A.3) for each $u \in X_{0}=\bigcup_{t>0} T(t)[X]$, but this and the remaining conditions of $(A)$-semigroups will be proved directly in the examples.

Let us now consider the Cauchy problem (A.2) with $d=1$ and

$$
P(\xi)=\left(\begin{array}{cc}
-\xi^{2}+i \xi^{4} & \xi^{q} \\
& -\xi^{2}+i \xi^{4}
\end{array}\right)
$$

then the order of equation $p=\max (4, q)$. Let $\Lambda(\xi)=\max _{j} \operatorname{Re} \lambda_{j}(\xi)$, where the $\lambda_{j}(\xi)$ are the eigenvalues of $P(\xi)$, then

$$
\Lambda(\xi) \leqq-\xi^{2},
$$

therefore the system $d \hat{u} / d t=P(\xi) \hat{u}$ is parabolic in the sense of Shilov with the exponent $h=2$. (See, for example, A. Friedman [2; Chap. 7, §6].) Since

$$
\exp (t P(\xi))=\left(\begin{array}{cc}
\exp \left(t\left(-\xi^{2}+i \xi^{4}\right)\right) & t \xi^{q} \exp \left(t\left(-\xi^{2}+i \xi^{4}\right)\right) \\
\exp \left(t\left(-\xi^{2}+i \xi^{4}\right)\right)
\end{array}\right)
$$

it is obvious $\sup _{\xi}|\exp (t P(\xi))|<\infty$ for $t>0$, so that the Cauchy problem is S.G. well-posed, and the type $\omega_{0}=0$.

The matrix theorem of Kreiss [5] shows that if $q=0,1$, or 2, then (A.2) is $\left(C_{0}\right)$-well-posed, and otherwise, it is not $\left(C_{0}\right)$-well-posed.

We shall show that (A.2) is $(0, A)$-well-posed if $q=3$ and 4 , and is not even 
(A)-well-posed if $q \geqq 5$. If $\operatorname{Re} \lambda>0$, then

$$
R(\lambda ; P(\xi))=\left(\begin{array}{ll}
1 /\left(\lambda+\xi^{2}-i \xi^{4}\right) & \xi^{q} /\left(\lambda+\xi^{2}-i \xi^{4}\right)^{2} \\
1 /\left(\lambda+\xi^{2}-i \xi^{4}\right)
\end{array}\right)
$$

for each $\xi$ and

$$
\|R(\lambda ; P)\|=\sup _{\xi}|R(\lambda ; P(\xi))|<\infty,
$$

if $q \leqq 8$. Therefore, if $q \leqq 8,\{\lambda ; \operatorname{Re} \lambda>0\}$ is contained in the resolvent set of $A$, and we have

$$
\|R(\lambda ; A)\| \leqq M, \quad \text { for } \operatorname{Re} \lambda>0
$$

This is the condition $\mathrm{b}$ ) of $(A)$-semigroups.

Next we shall show the condition a). Since $\{T(t)\}$ is strongly continuous for $t>0, D\left(A^{0}\right)$ is dense in $X_{0}=\bigcup_{t>0} T(t)[X]$ ([3; Theorem 10.3.1.]). It is obvious that the core $D \subset D\left(A^{0}\right)$ and $D$ is dense in $X$, hence $X_{0}$ is dense in $X$. Fix $\omega_{1}>\omega_{0}=0$, and take a $v \in X_{0}$, then there is a $t_{0}>0$ such that $v=T\left(t_{0}\right) u$, $u \in X$. But if $u \in D$, then $T\left(t_{0}\right) u \in D$ and we have the representation, by Lemma A, for $\operatorname{Re} \lambda>0$,

$$
(\lambda I-A)^{-1} T\left(t_{0}\right) u=\int_{0}^{\infty} \exp (-\lambda t) T(t) T\left(t_{0}\right) u d t .
$$

Now, $\operatorname{Re} \lambda>\omega_{1}$, then $\int_{0}^{\infty} \exp (-\lambda t) T\left(t+t_{0}\right) d t$ is a bounded linear operator on $X$, and $\int_{0}^{\infty} \exp (-\lambda t) T(t) T\left(t_{0}\right) u d t$ is well-defined for any $u \in X$. Therefore,

$$
\begin{aligned}
(\lambda I-A)^{-1} v=(\lambda I-A)^{-1} T\left(t_{0}\right) u & \\
& =\int_{0}^{\infty} \exp (-\lambda t) T(t) T\left(t_{0}\right) u d t=\int_{0}^{\infty} \exp (-\lambda t) T(t) v d t .
\end{aligned}
$$

Since $v \in X_{0}$ is arbitrary, this is the condition a).

Thus, in order to show $(A)$-well-posedness, it is sufficient to show the condition c), that is,

$$
\lim _{\lambda \rightarrow \infty} \lambda R(\lambda ; A) u=u, \quad \text { for each } u \in L^{2} .
$$

At first we shall show that $\sup _{\xi}\left|\xi^{a} /\left(\lambda+\xi^{2}-i \xi^{4}\right)^{2}\right|<\infty$ as $\lambda \rightarrow \infty$, if and only 
if $q \leqq 4$. But this is evident, because $\left|\xi^{a} /\left(\lambda+\xi^{2}-i \xi^{4}\right)^{2}\right|$ takes its maximum at the point $\xi^{4}=O(\lambda)$ as $\lambda \rightarrow \infty$. Thus we know $\|\lambda R(\lambda ; A)\| \leqq L$ as $\lambda \rightarrow \infty$, if $q \leqq 4$.

(A. 5) is evident for $u \in D$, dense in $L^{2}$, thus we obtain (A. 5) for any $u \in L^{2}$.

Finally we show that (A.2) with $q=3$ or 4 is actually $(0, A)$-well-posed. The proof depends on the Phillips criterion [8; Theorem 3.2] on $(0, A)$-well-posedness. It suffices to show that, if $u_{0} \in D(A)$, then

$$
T(t) u_{0} \rightarrow u_{0}, \quad \text { as } t \rightarrow 0,
$$

because we have shown that it is $(A)$-well-posed, and so the assumptions of the Phillips theorem are satisfied. (A.6) will be proved as follows:

As remarked before, $D(A)=\left\{\hat{u} \in L^{2} ; P(\xi) \hat{u} \in L^{2}\right\}$. Therefore, $u_{0}=\left(\begin{array}{l}u_{10} \\ u_{20}\end{array}\right)$ $\in D(A)$ if and only if

$$
P(\xi)\left(\begin{array}{l}
\hat{u}_{10} \\
\hat{u}_{20}
\end{array}\right)=\left(\begin{array}{c}
\left(-\xi^{2}+i \xi^{4}\right) \hat{u}_{10}+\xi^{4} \hat{u}_{20} \\
\left(-\xi^{2}+i \xi^{4}\right) \hat{u}_{20}
\end{array}\right) \in L^{2},
$$

that is, $\xi^{4} \hat{u}_{20}, \xi^{4} \hat{u}_{10} \in L^{2}$. Now

$$
\exp (t P(\xi)) \hat{u}_{0}=\left(\begin{array}{c}
\exp \left(t\left(-\xi^{2}+i \xi^{4}\right)\right) \hat{u}_{10}+t \xi^{4} \exp \left(t\left(-\xi^{2}+i \xi^{4}\right)\right) \hat{u}_{20} \\
\exp \left(t\left(-\xi^{2}+i \xi^{4}\right)\right) \hat{u}_{20}
\end{array}\right)
$$

and

$$
\begin{aligned}
& \exp \left(t\left(-\xi^{2}+i \xi^{4}\right)\right) \hat{u}_{20} \longrightarrow \hat{u}_{20}, \\
& \exp \left(t\left(-\xi^{2}+i \xi^{4}\right)\right) \hat{u}_{10} \longrightarrow \hat{u}_{10},
\end{aligned}
$$

are evident. Since $\xi^{4} \hat{u}_{20} \in L^{2}$, it is also evident that

$$
t \xi^{4} \exp \left(t\left(-\xi^{2}+i \xi^{4}\right)\right) \hat{u}_{20} \longrightarrow 0, \quad \text { as } t \rightarrow 0 \text {. }
$$

Thus we obtain (A. 6), and the proof is completed.

As corollaries, we know that

$$
\begin{gathered}
\left.\int_{0}^{1} \| \exp ^{\prime} t P(\xi)\right) \hat{u}_{0}(\xi) \| d t<\infty, \quad \text { for any } u_{0} \in L^{2}, \\
R(\lambda ; P) u_{0}=\int_{0}^{\infty} \exp (-\lambda t) T(t) u_{0} d t, \quad \text { for any } u_{0} \in L^{2},
\end{gathered}
$$


by the properties of $(0, A)$-semi-groups.

REMARK. In the above example, if $q=3$, then the Cauchy problem (A. 2) is $(1, A)$-well-posed. In fact, it is sufficient to show that $\int_{0}^{1}\|T(t)\| d t<\infty$, or

$$
\int_{0}^{1} \sup _{\xi}\left|t \xi^{3} \exp \left(t\left(-\xi^{2}+i \xi^{4}\right)\right)\right| d t<\infty .
$$

But this is shown by the fact that the integrand takes the maximum at the point of $t \xi^{2}=3 / 2$, therefore $\sup _{\xi}\left|t \xi^{3} \exp \left(t\left(-\xi^{2}+i \xi^{4}\right)\right)\right|=O\left(1 / t^{1 / 2}\right)$ as $t \rightarrow 0$.

\title{
REFERENCES
}

[1] G. BIRKHOFF AND R.S. VARGA, Discretization errors for well-set Cauchy problems. 1, J. Math. and Phys., 44(1965), 1-23.

[2] A. FRIEDMAN, Generalized functions and partial differential equations, Prentice-Hall, Englewood Cliffs, N. J. 1963.

[ 3 ] E. Hille AND R.S. PHIllips, Functional analysis and semi-groups, A. M.S. Colloq. Vol. 31, (revised ed.) (1957).

[4] T. KATO, Perturbation theory for linear operators, Springer, Berlin-Heidelberg-New York, (1966).

[5] H.O. KREISS, Ueber Matrizen die beschränkte Halbgruppe erzeugen, Math. Scand., 7 (1959), 71-80.

[6] I. MrYADERA, Perturbation theory for semigroups of operators, (in Japanese) Sugaku, 20(1968), 14-25.

[ 7 ] S. OHARU AND H. SUNOUCHI, On the convergence of semigroups of linear operators, J. Funct. Anal., 6(1970)

[ 8 ] R.S. PHILliPS, A note on the abstract Cauchy problem, Proc. Nat. Acad. Sci. U.S. A., 40(1954), 244-248.

[9] H.F. TROTTER, Approximation of semi-groups of operators, Pacific J. Math., 8(1958), 887-919.

\author{
DEPARTMENT OF MATHEMATICS \\ WASEDA UNIVERSITY \\ TOKYO, JAPAN
}

